\title{
The Early Mother-to-Child Bond and Its Unique Prospective Contribution to Child Behavior Evaluated by Mothers and Teachers
}

\author{
Anna Fuchs ${ }^{a, b} \quad$ Eva Möhler ${ }^{b} \quad$ Corinna Reck $^{c, d}$ Franz Resch $^{b} \quad$ Michael Kaess $^{a, b}$ \\ a Section for Translational Psychobiology in Child and Adolescent Psychiatry, and ${ }^{b}$ Department of Child and \\ Adolescent Psychiatry, Centre for Psychosocial Medicine, and ' Department of Psychiatry, University of Heidelberg, \\ Heidelberg, and ${ }^{\mathrm{d}}$ Department of Child and Adolescent Clinical Psychology and Counseling Psychology, Faculty of \\ Psychology and Educational Science, Ludwig Maximilian University, Munich Germany
}

\section{Key Words}

Bonding $\cdot$ Child behavior problems $\cdot$ Maternal psychopathology · Child psychopathology · Mother-child relationship · Mother-infant bond - Longitudinal studies

\begin{abstract}
Maternal bonding has been described as the quality of the affective tie from a mother to her infant. This early bond's mental components and its longitudinal impact on child outcome have been markedly understudied. Although most researchers assume impaired maternal bonding to have a negative impact on child development, there is a lack of prospective studies evaluating this hypothesis. Since maternal mental health problems may negatively affect both bonding quality and child development, it is still to be determined whether there is a unique contribution of bonding quality to child behavior problems over and above maternal psychopathology. We examined a community sample of 101 mother-child dyads at the child's age of 2 weeks (t1) and 6 weeks (t2), 4 months ( $\mathrm{t} 3$ ), 14 months ( $\mathrm{t} 4)$, and 5.5 years ( $\mathrm{t} 5$ ). Maternal bonding and psychopathology were assessed at time points $\mathrm{t} 1-\mathrm{t} 4 \mathrm{using}$ the Postpartum Bonding Questionnaire (PBQ-16) and the Symptom Checklist Revised (SCL 90-R). Child behavior problems were rated in a multi-informant design by mothers and teachers at $t 5$ using the Strengths and
\end{abstract}

Difficulties Questionnaire (SDQ). In the case of maternal judgment of child behavior problems, bonding at 14 months (t4) proved to be a significant predictor $(\beta=0.30 ; p=0.011)$. Teacher-rated child behavior problems were significantly predicted by maternal bonding at 2 weeks $(\mathrm{t} 1 ; \beta=0.48 ; \mathrm{p}=$ 0.025). Our results indicate a prospective influence of the early mother-infant bond on child development and underline the unique contribution of bonding quality to child behavior problems over and above the impact of maternal psychopathology in a community sample.

(c) 2016 S. Karger AG, Basel

\section{Introduction}

Maternal bonding has been described as the quality of the affective tie from a mother to her infant, complementing the attachment relationship that the infant develops over the course of the first year [1]. Since John Bowlby's and Mary Ainsworths' early work on attachment (Bowlby [2], 1969; Ainsworth et al. [3], 1978), the formation of a durable bond between mother and child, and especially its behavioral components, has been studied extensively. However, although unique to the human adult, far less is known about the mental aspects of the formation process [4]. From pregnancy onwards, maternal fear and reward

\section{KARGER}

E-Mail karger@karger.com

www.karger.com/psp
(C) 2016 S. Karger AG, Basel

0254-4962/16/0494-0211\$39.50/0
Anna Fuchs, Dipl. Psych.

Department of Child and Adolescent Psychiatry Centre for Psychosocial Medicine, University of Heidelberg

Blumenstrasse 8, DE-69115 Heidelberg (Germany)

E-Mail anna.fuchs@med.uni-heidelberg.de 
systems include mental aspects such as preoccupation with the infant's well-being or attachment representations of the child and the shared future, indicating an organizing role of cognition [5]. Oxytocin, a neuropeptide affiliated with social behavior in mammals, has been shown to be associated with attachment-related thoughts $[4,5]$. Therefore, in addition to the study of maternal behavior, examining a mother's cognitions and reported feelings about her child enables researchers to grasp a comprehensive picture of the development of human affiliation.

Compared to the fields of child attachment and behavioral mother-child interaction, the early bond from mother to infant and its correlates, developmental pathways and impact on child outcome, have been markedly understudied [6-8]. Although most researchers assume failed mother-to-child bonding to exert a negative influence on the emotional and behavioral development of the child, there is a significant lack of studies evaluating this hypothesis [9]. Only one study so far has tried to shed light on the association between maternal feelings of attachment and child outcome: Mason et al. [10] (2011) examined a low-income urban sample of 232 first-time mothers and found maternal bonding at infant age of 2 months to be associated with maternal reports of their social-emotional development at 6 months.

Bonding-related representations and maternal feelings about their child seem to be highly sensitive to risk conditions, such as maternal psychopathology and premature birth [4]. Maternal mental health has been shown to exert a detrimental influence on parental bonding [11, 12]. Parents with low bonding patterns have been characterized by increased anxiety and parenting stress [7], higher levels of depression or PTSD [13-16], and higher scores of overall psychopathology [12]. It is notable, however, that women who do not suffer from mental disorders also seem to experience poor bonding [17]. Even in subclinical samples, there is a range of reported bonding quality [14, 15]. In addition, O'Higgins et al. [9] (2013) emphasize that maternal psychopathology and bonding, although closely related, still represent independent factors following different courses of development and possibly exerting differential influences on child outcome. While it is clear that maternal psychopathology poses a risk factor for child development $[18,19]$, it is still to be determined whether there is a unique contribution of bonding quality to child behavior problems.

In clinical practice, multi-informant sources on child behavior form the basis of a valid diagnostic routine. Given that children's behavior may be context specific, depending on the situational context and the person they are interacting with, multiple informants enable clinicians to obtain a comprehensive picture of a child's behavioral patterns [20-22]. Additionally, bearing in mind that parental characteristics and parental psychopathology in particular might lead to biased perceptions of child behavior $[23,24]$, the interpretation of results might be limited. However, due to limited resources and cooperation of additional sources other than parents, most research - such as the only study examining associations between maternal bonding and child outcome [10] - has been relying on a single-informant design.

In conclusion, although of importance, maternal thoughts and feelings as indicators of the early mother-tochild bond represent a neglected area of research. In particular, the association between early bonding and later child behavior problems has been markedly understudied, with only one study examining the relationship between bonding and maternal report of social-emotional development at 6 months of infant age [10]. As maternal psychopathology has been shown to impact maternal bonding and child behavior problems, but at the same time differences in bonding have been found in nonclinical samples, we examined whether maternal bonding would exert an impact on child outcome even after the influence of psychopathology was controlled for. We therefore expanded the insights gathered by Mason et al. [10] (2011) by examining a low-risk sample over a longer period of time, using multiple measurements and a multi-informant approach. We expected high levels of impaired bonding to be associated with high levels of child behavior problems.

\section{Methods}

\section{Study Procedure}

Before potential participants were approached, the study was approved by the Ethics Committee of the Faculty of Medicine, University of Heidelberg. Written informed consent was obtained from all mothers before the study. Subsequently, mother-child dyads were seen in the laboratory at the child's age of 2 weeks (t1), 6 weeks (t2), 4 months (t3), 14 months (t4), and 5.5 years (t5). Socioeconomic status was documented at the first and last assessment, and infant health status was assessed at every visit. Maternal general psychopathology was measured at all time points, postpartum bonding was measured at the first four time points, and child behavior was assessed at $\mathrm{t} 5$.

\section{Participant Recruitment}

The sample of this study consisted of a volunteer sample of 102 healthy Caucasian mothers and their infants after singleton pregnancies. Mothers were recruited in the four major local obstetric units over a period of 9 months beginning in June 2002. Eligibility criteria were full-term deliveries and infant weight $>2,500 \mathrm{~g}$, APGAR scores $>7$, and good health of the baby as documented by the 
Table 1. Mean \pm SD for bonding, maternal psychopathology, and child outcome

\begin{tabular}{llllll}
\hline & 2 weeks & 4 weeks & 4 months & 14 months & 5.5 years \\
\hline Bonding & $8.66 \pm 5.28$ & $9.09 \pm 6.26$ & $7.04 \pm 4.29$ & $8.45 \pm 4.52$ & \\
GSI & $0.29 \pm 0.23$ & $0.22 \pm 0.22$ & $0.22 \pm 0.23$ & $0.24 \pm 0.27$ & \\
SDQ mother & & & & & $11.47 \pm 2.94$ \\
SDQ teacher & & & & $10.97 \pm 2.23$ \\
\hline
\end{tabular}

first, second, and third postnatal examination. Exclusion criteria were the inability to speak and read the German language, use of drugs or medications posing a risk to the fetus, and excessive smoking ( $>5$ cigarettes/day) or alcohol consumption during pregnancy.

At $\mathrm{t} 1$, the participating women were between ages 19 and 45 years $($ mean $=33.3)$. Mean infant weight was $3,497 \mathrm{~g}(\mathrm{SD}=3.8$; range: $2,520-4,500)$. Overall, $45.0 \%$ of the infants were girls, $51.5 \%$ were firstborn, $35.6 \%$ had one sibling, and $12.9 \%$ had two or more siblings. Compared to the general population, the sample was highly educated: $24.8 \%$ of the mothers had achieved intermediate secondary education, $19.8 \%$ had university entrance qualification, and $55.4 \%$ of the mothers had achieved a university degree. All mothers were in a partnership with the infant's father.

All 101 participating women had agreed to be contacted for phase two of the study and were therefore recontacted. Out of the 101 mothers, 89 (88\%) agreed to take part in the reassessment. Comparing data from the first phase of the study, women not participating in the follow-up did not differ from the 89 remaining mothers regarding partnership status (all mothers were in a partnership), education $\left(\chi_{2,101}^{2}=2.11, \mathrm{p}=0.349\right)$, child's gender $\left(\chi_{2}^{2}\right.$, $\left.{ }_{101}=0.69, \mathrm{p}=0.540\right)$, child's birth weight $\left(\mathrm{t}_{99}=-0.25 ; \mathrm{p}=0.802\right)$, or number of children $\left(\mathrm{t}_{99}=0.67 ; \mathrm{p}=0.508\right)$. Their mean age, however, was significantly lower $\left(\mathrm{t}_{99}=2.19 ; \mathrm{p}=0.031\right)$, with a mean age of 30.9 years at the time of the first assessment compared to 33.6 years. The final sample of the second study phase comprised 89 mothers and their children (mean age $=5.7$ years; range: $5.5-6.7$ ). Most mothers were still in a relationship with the child's father (79.2\%), 6.9\% were separated, and 1 mother was widowed. The children spent a mean of $28.9 \mathrm{~h}$ (SD: 7.3) per week in day care.

\section{Measures}

Maternal bonding was measured using the Postpartum Bonding Questionnaire (PBQ) [25]. Earlier work based on this study used a translated version of this 25 -item self-report instrument, which is based on 4 factors [14]. However, a recent validation study did not confirm the original 4-factor structure for the German translation but suggested a 16-item single-factor solution labeled as 'bonding impairment', with higher scores indicating lower bonding [26]. As for our sample, the PBQ-16 total score showed good internal consistency for each measurement. Cronbach's alpha values were $\alpha=0.77(\mathrm{t} 1), \alpha=0.85(\mathrm{t} 2), \alpha=0.70(\mathrm{t} 3)$, and $\alpha=$ $0.74(\mathrm{t} 4)$.

We used the German version of the Symptom Checklist 90-Revised (SCL-90-R) as a broadly defined indicator for maternal mental health [27]. The SCL-90-R is a 90 -item self-report inventory measuring psychological distress in nine dimensions and three global scales. The global severity index (GSI), obtained by averaging the scores over the 90 items, is considered to be the best indicator of the current degree of psychological distress [27]. Its use has been reinforced by recent validation studies [28].

Early Mother-to-Child Bond and Child Behavior Problems
Child behavior problems were assessed by the German version of the Strengths and Difficulties Questionnaire (SDQ) [29, 30]. The SDQ shows strong psychometric properties in school-age as well as preschool-age samples $[21,31]$. It is recommended as a valid screening instrument to assess children's behavior [32]. Children with higher total difficulty scores exhibit higher levels of psychopathology as judged by the prevalence of clinical disorder [33].

\section{Data Analysis}

For statistical analysis, we applied correlation analysis as well as single and multiple regression analysis. To calculate the effect of maternal bonding and at the same time control for the impact of maternal psychopathology, we used residuals generated by regressing PBQ variables on SCL-90 GSI variables. Those adjusted PBQ variables represent what is left in the variation of the PBQ after GSI has been partialled out. Correspondingly, they contain the variation of maternal bonding that is independent of maternal psychopathology. We used the Statistical Package for Social Sciences (IBM SPSS v. 22.0) for all the analyses conducted in this study.

\section{Results}

There were no significant associations between sociodemographic variables and the children's SDQ scores except for hours spent in day care per week (t5) and maternal judgment of child behavior problems (Pearson's $r=0.32 ; \mathrm{p}<0.01)$. Similarly, the associations between sociodemographic variables and maternal PBQ scores proved to be nonsignificant except for hours spent in day care per week (Pearson's $r ; \mathrm{t} 1: r=0.28, \mathrm{p}=0.017 ; \mathrm{t} 2: r=$ $0.30, \mathrm{p}<0.01 ; \mathrm{t} 3: r=0.32, \mathrm{p}<0.01 ; \mathrm{t} 4: r=0.34, \mathrm{p}<0.01)$. In order to control for the confounding effect of time in day care, the variable was included in all analysis. Maternal and teacher ratings of child behavior problems were associated only at trend level (two-way mixed singlemeasures intraclass correlation coefficient $=0.189 ; \mathrm{p}=$ $0.05)$. For the descriptive statistics of the main variables, see table 1 .

To test our hypothesis of an association between maternal bonding and child behavior problems independent from maternal psychopathology, we first conducted simple regression analyses by regressing the SDQ variables on the PBQ-adjusted variables. In a second step, we calculated multiple regression models. Results show signifi- 
Table 2. Adjusted maternal bonding predicting child behavior problems (maternal rating)

\begin{tabular}{lcccc}
\hline & B & SE & $\beta$ & $\beta$ sig \\
\hline Explanatory variable & & & & \\
Day care & 0.13 & 0.04 & 0.32 & $<0.01$ \\
PBQ adj 1 & 0.08 & 0.08 & 0.14 & 0.274 \\
PBQ adj 2 & 0.11 & 0.06 & 0.21 & 0.065 \\
PBQ adj 3 & 0.07 & 0.08 & 0.10 & 0.393 \\
PBQ adj 4 & 0.17 & 0.07 & 0.27 & 0.014 \\
Multiple regression model & & & & \\
Day care & 0.07 & 0.06 & 0.16 & 0.247 \\
PBQ adj 1 & 0.04 & 0.11 & 0.06 & 0.753 \\
PBQ adj 2 & 0.02 & 0.12 & 0.03 & 0.882 \\
PBQ adj 3 & -0.26 & 0.15 & -0.39 & 0.077 \\
PBQ adj 4 & 0.30 & 0.12 & 0.51 & 0.011 \\
Final model & $\mathrm{R}^{2}=0.185 ; \mathrm{p}=0.041$ & \\
\hline
\end{tabular}

PBQ adj = PBQ adjusted for maternal level of psychopathology; day care $=$ hours in day care per week; $\alpha=0.05$.

Table 3. Adjusted maternal bonding predicting child behavior problems (teacher rating)

\begin{tabular}{lcccc}
\hline & B & SE & $\beta$ & $\beta$ sig \\
\hline Explanatory variable & & & & \\
$\quad$ Day care & 0.03 & 0.03 & 0.11 & 0.356 \\
PBQ adj 1 & 0.12 & 0.06 & 0.26 & 0.042 \\
PBQ adj 2 & 0.02 & 0.05 & 0.05 & 0.701 \\
PBQ adj 3 & 0.06 & 0.06 & 0.12 & 0.314 \\
PBQ adj 4 & 0.07 & 0.06 & 0.14 & 0.223 \\
Multiple regression model & & & & \\
Day care & -0.01 & 0.05 & -0.03 & 0.839 \\
PBQ adj 1 & 0.22 & 0.10 & 0.48 & 0.025 \\
PBQ adj 2 & -0.18 & 0.10 & -0.43 & 0.084 \\
PBQ adj 3 & 0.02 & 0.13 & 0.04 & 0.878 \\
PBQ adj 4 & 0.08 & 0.10 & 0.17 & 0.428 \\
Final model & $\mathrm{R}^{2}=0.123 ; \mathrm{p}=0.210$ & \\
\end{tabular}

PBQ adj = PBQ adjusted for maternal level of psychopathology; day care $=$ hours in day care per week; $\alpha=0.05$.

cant associations between bonding scores and child behavior problems, but mother (table 2) and teacher (table 3) ratings were associated at different measurement times. As for maternal judgment of child behavior problems, maternal bonding at 14 months ( $\mathrm{t} 4)$ proved to be a significant predictor in the simple $(\beta=0.27 ; \mathrm{p}=0.014)$ as well as in the multiple regression model $(\beta=0.30 ; p=$ 0.011 ). Regarding teacher-rated child behavior problems, only maternal bonding at 2 weeks ( $\mathrm{t} 1)$ reached significance, again in the simple $(\beta=0.26 ; p=0.042)$ and multiple $(\beta=0.48 ; p=0.025)$ regression models. The overall model of maternal bonding predicting child behavior problems reached significance only in the case of maternal rating $\left(R^{2}=0.185 ; p=0.041\right)$. Hours spent in day care per week proved to be a significant predictor for maternal judgement of child behavior, and only in the case of simple regression $(\beta=0.32 ; p<0.01)$. In the overall model including all PBQ variables, hours spent in day care did not significantly predict child behavior problems.

\section{Discussion}

Our results confirm the postulated prospective influence of the early mother-infant bond on child development. We found the quality of early maternal bonding to exert a significant impact on child behavior problems at the age of 5-6 years even after the influence of maternal psychopathology level had been partialled out. Low levels of bonding were associated with high levels of child behavior problems rated by mother and teacher. These results confirm the hypothesized importance of the early bond from mother to child with regard to child development, thereby closing a gap in the literature. As stated by Feldman et al. [4] (1999), maternal bonding includes not only behavioral but also mental aspects such as attachment representations of the child and the shared future. Considering attachment as incorporating both components may promote a sophisticated understanding of the formation of human affiliation $[3,4]$.

Our results showed significant associations between bonding variables and maternal as well as teacher judgement of child behavior problems. However, they suggest different periods to render the effect. As for teacher rating of behavior, the time immediately after birth appears to play a role in child development. Maternal rating, however, was shown to be influenced mainly by their reported bonding at the child's age of 14 months. This raises the question of whether maternal thoughts and feelings about the infant might be increasingly influenced by the child's behavior and developing personality, which could be reflected in their bonding scores and lead to a higher correlation of bonding and behavior judgment. Between the age of 4 and 14 months lies the onset of child locomotion, an important milestone in child development, changing the relation between infant and its environment dramatically [34]. The child's new autonomy requires mothers to exhibit alertness to a larger degree, but simultaneously, there is now proactive proximity seeking on part of the child, an important 
factor in the formation of attachment $[3,35]$. It could be hypothesized that maternal feelings and thoughts on their older infant are affected by the child's developing mobility and temperament, and therefore show high correlations with reported child behavior problems later on. Contrary to this, maternal thoughts and feelings about her infant during the first 2 weeks after birth might capture her bonding quality more independently from child input. The significant association between maternal bonding level at 2 weeks and teacher rating of behavior problems even 5-6 years later underlines the hypothesis of Brockington [36] (2004) of bonding being the most important psychological process following childbirth. However, even though the assessment at 2 weeks was a significant predictor, and there was an association at trend level at 4 months, we did not find associations in terms of later measurements. In addition, the overall model did not reach significance, apart from the model calculating associations with maternal rating of child behavior. Given the time span between the assessments and the independence of teacher rating from mother-child interactional influence, the predictive value of the 2 -week assessment is still noteworthy. Even though the idea of a rigorous 'sensitive period' defining the first postpartum days or even hours and being critical in the formation of human bonds is outdated $[37,38]$, the birth of a child is still a life transition period for the mother, requiring significant alteration in customary life patterns [38]. Some mothers seem to have difficulties adapting and building an emotional bond with their child even without the additive strain of suffering from psychopathology $[13,26]$. Our results indicate that these difficulties might be associated with later child behavior problems in kindergarten age.

\section{Strengths and Limitations}

This is the first study to examine the impact of the quality of maternal bonding on child behavior problems in a multi-informant design using a longitudinal approach. A first limitation might relate to the representativeness of the sample. Results drawn from our community sample might not be generalized to the general population, as our sample was drawn from a small university town, thus including a high percentage of middle-to-upper-class families and well-educated parents. However, the investigation of a low-risk Caucasian population is in line with the evidence reported by Mason et al. [10] (2011) from a high-risk and racially diverse urban sample. As another limitation, we applied questionnaires for the assessment of the constructs of interest, so we cannot rule out the possibility of response bias. A standardized interview assessing maternal thoughts and feelings with respect to bonding in the German language is still missing, and as maternal mental state is the subject of investigation, self-rating questionnaires are the obvious choice. To be able to cover a comprehensive understanding of child behavior in two contexts and to reduce the risk for bias, we collected both maternal and teacher ratings. We did not include paternal measures, an aspect that should be taken on in future studies, as the mother-child relationship is not the only relationship to play a central role in the infant's development $[39,40]$.

\section{Conclusion}

Our results give evidence of a unique prospective contribution of the quality of maternal bonding to child behavior problems over and above the impact of maternal psychopathology. Even though we did not first and foremost examine mothers diagnosed with a psychiatric disorder, our findings might still be relevant for clinical practice: it might not be sufficient to simply target maternal psychopathology to reduce the risk for child behavior difficulties. This would be in line with the reasoning of Forman et al. [41] (2007), who found that simply reducing symptoms of depression did not improve child outcome. Instead, complementary interventions addressing psychopathology as well as the mother-child relationship might be the optimal approach. As recent results indicate prevention programs targeting attachment [for an overview, see 42], or even simple interventions such as baby massage, to be effective in improving the mother-child interaction [43, 44], a broad implementation of these programs might be worthwhile not only in clinical but also in nonclinical dyads.

\section{Acknowledgment}

The authors would like to thank the German Research Foundation (DFG, Mo 978/1-1/2) as well as the German Federal Ministry of Education and Research for their financial support (grant 01KR1207A) of the present study.

References

Psychopathology 2016;49:211-216
Solomon J, George C: Defining the caregiving system: toward a theory of caregiving. Infant Ment Health J 1996;17:183-197.

2 Bowlby J: Attachment and Loss: Attachment. New York, Basic Books, vol 1, 1969.

-3 Ainsworth MD, Blehar MC, Waters E, Wall S: Patterns of Attachment: A Psychological Study of the Strange Situation. Hillsdale, Erlbaum, 1978. 
4 Feldman R, Weller A, Leckman JF, Kvint J, Eidelman AI: The nature of the mother's tie to her infant: maternal bonding under conditions of proximity, separation, and potential loss. J Child Psychol Psychiatry 1999;40:929939.

5 Feldman R, Weller A, Zagoory-Sharon O, Levine A: Evidence for a neuroendocrinological foundation of human affiliation plasma oxytocin levels across pregnancy and the postpartum period predict mother-infant bonding. Psychol Sci 2007;18:965-970.

-6 Redshaw M, Martin C: Babies, 'bonding' and ideas about parental 'attachment'. J Reprod Infant Psychol 2013;31:219-221.

-7 De Cock ES, Henrichs J, Vreeswijk CM, Maas AJ, Rijk CH, van Bakel HJ: Continuous feelings of love? The parental bond from pregnancy to toddlerhood. J Fam Psychol 2016;30: 125-134.

-8 Hall RA, Hoffenkamp HN, Tooten A, Braeken J, Vingerhoets AJ, Van Bakel HJ: Childrearing history and emotional bonding in parents of preterm and full-term infants. J Child Fam Stud 2014;24:1715-1726.

-9 O'Higgins M, Roberts ISJ, Glover V, Taylor A: Mother-child bonding at 1 year; associations with symptoms of postnatal depression and bonding in the first few weeks. Arch Womens Ment Health 2013;16:381-389.

10 Mason ZS, Briggs RD, Silver EJ: Maternal attachment feelings mediate between maternal reports of depression, infant social-emotional development, and parenting stress. J Reprod Infant Psychol 2011;29:382-394.

11 Kerstis B, Aarts C, Tillman C, Persson H, Engström G, Edlund B, Skalkidou A: Association between parental depressive symptoms and impaired bonding with the infant. Arch Womens Ment Health 2014;1-8.

12 Örün E, Yalçın SS, Mutlu B: Relations of maternal psychopathologies, social-obstetrical factors and mother-infant bonding at 2-month postpartum: a sample of Turkish mothers. World J Pediatr 2013;9:350-355.

13 Muzik M, Bocknek EL, Broderick A, Richardson P, Rosenblum KL, Thelen K, Seng JS: Mother-infant bonding impairment across the first 6 months postpartum: the primacy of psychopathology in women with childhood abuse and neglect histories. Arch Womens Ment Health 2013;16:29-38.

-14 Moehler E, Brunner R, Wiebel A, Reck C, Resch F: Maternal depressive symptoms in the postnatal period are associated with longterm impairment of mother-child bonding. Arch Womens Ment Health 2006;9:273-278.

15 Tietz A, Zietlow A-L, Reck C: Maternal bonding in mothers with postpartum anxiety disorder: the crucial role of subclinical depressive symptoms and maternal avoidance behaviour. Arch Womens Ment Health 2014;17: 433-442.

16 Wittkowski A, Wieck A, Mann S: An evaluation of two bonding questionnaires: a comparison of the Mother-to-Infant Bonding Scale with the Postpartum Bonding Ques- tionnaire in a sample of primiparous mothers. Arch Womens Ment Health 2007;10:171175.

17 Righetti-Veltema M, Conne-Perréard E, Bousquet A, Manzano J: Postpartum depression and mother-infant relationship at 3 months old. J Affect Disord 2002;70:291306.

18 Betts KS, Williams GM, Najman JM, Alati R: The relationship between maternal depressive, anxious, and stress symptoms during pregnancy and adult offspring behavioral and emotional problems. Depress Anxiety 2015; 32:82-90.

19 Goodman SH, Rouse MH, Connell AM, Broth MR, Hall CM, Heyward D: Maternal depression and child psychopathology: a meta-analytic review. Clin Child Fam Psychol Rev 2011;14:1-27.

20 De Los Reyes A, Kazdin AE: Informant discrepancies in the assessment of childhood psychopathology: a critical review, theoretical framework, and recommendations for further study. Psychol Bull 2005;131:483.

-21 Korsch F, Petermann F: Agreement between parents and teachers on preschool children's behavior in a clinical sample with externalizing behavioral problems. Child Psychiatry Hum Dev 2014;45:617-627.

22 Offord DR, Boyle MH, Racine Y, Szatmari P, Fleming JE, Sanford M, Lipman EL: Integrating assessment data from multiple informants. J Am Acad Child Adolesc Psychiatry 1996;35:1078-1085.

23 Kroes G, Veerman JW, De Bruyn EE: Bias in parental reports? Maternal psychopathology and the reporting of problem behavior in clinic-referred children. Eur J Psychol Assess 2003;19:195.

24 Mulvaney MK, Mebert CJ, Flint J: Parental affect and childrearing beliefs uniquely predict mothers' and fathers' ratings of children's behavior problems. J Appl Dev Psychol 2007;28: 445-457.

25 Brockington IF, Oates J, George S, Turner D, Vostanis P, Sullivan M, Murdoch C: A screening questionnaire for mother-infant bonding disorders. Arch Womens Ment Health 2001; 3:133-140.

26 Reck C, Klier CM, Pabst K, Stehle E, Steffenelli U, Struben K, Backenstrass M: The German version of the Postpartum Bonding Instrument: psychometric properties and association with postpartum depression. Arch Womens Ment Health 2006;9:265-271.

27 Franke GH, Derogatis LR: SCL-90-R: Symptom-Checkliste von LR Derogatis: deutsche Version: Manual. Weinheim, Beltz, 2002.

28 Urbán R, Kun B, Farkas J, Paksi B, Kökönyei G, Unoka Z, Demetrovics Z: Bifactor structural model of symptom checklists: SCL-90-R and Brief Symptom Inventory (BSI) in a nonclinical community sample. Psychiatry Res 2014;216:146-154.

29 Goodman R: The Strengths and Difficulties Questionnaire: a research note. J Child Psychol Psychiatry 1997;38:581-586.
30 Klasen $\mathrm{H}$, Woerner W, Rothenberger A, Goodman R: German version of the Strength and Difficulties Questionnaire (SDQ-German) - overview and evaluation of initial validation and normative results. Prax Kinderpsychol Kinderpsychiatr 2003;52:491-502.

- 31 Stone LL, Otten R, Engels RC, Vermulst AA, Janssens JM: Psychometric properties of the parent and teacher versions of the strengths and difficulties questionnaire for 4-to 12-yearolds: a review. Clin Child Fam Psychol Rev 2010;13:254-274.

32 Petermann U, Petermann F, Schreyer I: The German Strengths and Difficulties Questionnaire (SDQ): validity of the teacher version for preschoolers. Eur J Psychol Assess 2010; 26:256-262.

33 Goodman A, Goodman R: Strengths and Difficulties Questionnaire as a dimensional measure of child mental health. J Am Acad Child Adolesc Psychiatry 2009;48:400-403.

-34 Anderson DI, Campos JJ, Witherington DC, Dahl A, Rivera M, He M, Barbu-Roth M: The role of locomotion in psychological development. Front Psychol 2013;4:440.

35 Campos JJ, Anderson DI, Barbu-Roth MA, Hubbard EM, Hertenstein MJ, Witherington D: Travel broadens the mind. Infancy 2000;1: 149-219.

36 Brockington I: Postpartum psychiatric disorders. Lancet 2004;363:303-310.

37 Bornstein MH: Sensitive periods in development: structural characteristics and causal interpretations. Psychol Bull 1989;105:179.

38 Myers B J: Mother-infant bonding: the status of this critical-period hypothesis. Dev Rev 1984;4:240-274.

39 Cohen D, Cassel RS, Saint-Georges C, Mahdhaoui A, Laznik M-C, Apicella F, Chetouani M: Do parentese prosody and fathers' involvement in interacting facilitate social interaction in infants who later develop autism? PLoS One 2013;8:e61402.

40 Ramchandani PG, Domoney J, Sethna V, Psychogiou L, Vlachos H, Murray L: Do early father-infant interactions predict the onset of externalising behaviours in young children? Findings from a longitudinal cohort study. J Child Psychol Psychiatry 2013;54:56--64.

41 Forman DR, O'Hara MW, Stuart S, Gorman LL, Larsen KE, Coy KC: Effective treatment for postpartum depression is not sufficient to improve the developing mother-child relationship. Dev Psychopathol 2007;19:585-602.

42 Dozier M, Roben CK: Attachment-related preventive interventions; in Simpson JA, Rholes WS (eds): Attachment Theory and Research: New Directions and Emerging Themes. New York, Guildford Press, 2015, p 374.

43 Gürol A, Polat S: The effects of baby massage on attachment between mother and their infants. Asian Nurs Res 2012;6:35-41.

44 O'Higgins M, St James Roberts I, Glover V: Postnatal depression and mother and infant outcomes after infant massage. J Affect Disord 2008;109:189-192. 